\author{
K. Michael A. Welch \\ Susan M. Bowyer \\ Sheena K. Aurora \\ John E. Moran \\ Norman Tepley
}

\section{Visual stress-induced migraine aura compared to spontaneous aura studied by magnetoencephalography}

K.M.A. Welch ( $₫)$

University of Kansas Medical Center, 3901

Rainbow Blvd., 6002 Wescoe

Kansas City, Kansas 66160-7702, USA

e-mail: kwelch2@kumc.edu

Tel.: +1-913-588-5436

Fax: +1-913-588-5444

S.M. Bowyer • J.E. Moran • N. Tepley Department of Neurology, Henry Ford Health System, Neuromagnetism Laboratory,

Detroit, Michigan, U.S.A.

S.K. Aurora

Swedish Neurological Institute, Seattle, Washington, USA

This paper has been presented in part at the Proceedings of the International Headache Society, Barcelona 1999. Extended results of the study are to be published in Annals of Neurology.

\begin{abstract}
DC MEG shifts, similar and complex in waveform, were observed in visually induced migraine with aura patients similar to spontaneous aura but not controls. Multiple cortical areas were activated in visually induced and spontaneous aura patients. In normal subjects activation was only observed in the primary visual cortex. Results support a spreading depression-like neuroelectric event as the basis of migraine aura that can arise spontaneously or be visually triggered in widespread regions of hyperexcitable occipital cortex.
\end{abstract}

Key words DC MEG • Migraine with aura $\cdot$ Migraine without aura . Occipital hyperexcitability

\section{Introduction}

Spreading cortical depression of Leao (SCD) is thought to be the underlying mechanism of migraine aura [1-4]. During experimental SCD, excitable neurons in the cortical surface fire and become electrically silent for up to 30 minutes [3-6]. The band of synchronous depolarization of neurons propagates across the cortical surface at a rate of 3-7 mm/min [1, 3, 4]. The slowly spreading activation followed by suppression may be the basis of the slow march of positive followed by negative neurological symptoms of migraine aura [2].
Magnetoencephalography (MEG), a noninvasive technique used for mapping magnetic fields arising from cortical electrical activity, is better suited to detect spreading cortical depression (SCD) than conventional electroencephalography (EEG) [7], and has been used in experimental animals and patients to study mechanisms of migraine [8-11]. Characteristic MEG waveforms arising during SCD have been established in animal models $[12,13]$. This study investigates MEG waveforms arising during spontaneous and visually induced migraine aura in humans, to ascertain whether signals occur indicative of SCD, and to further establish an association between SCD and migraine. 


\section{Subjects and methods}

Subjects

DC MEG recordings were measured during visual stimulation of the occipital cortex in eight migraine with aura patients, in four migraine patients during and after the onset of spontaneous with aura patients migraine with aura, and in six normal controls with no history of migraine headache. Migraine patients were diagnosed by two of the authors (SA, KMAW) based on the International Headache Society (IHS) criteria [14]. The migraine with aura patients had a history of migraine attacks triggered by visual stimuli (bright light, flashing lights, strobe lights). No patients were taking preventive medications and none suffered a migraine attack within the preceding seven days. All subjects gave written informed consent prior to study. The Institutional Review Board of Henry Ford Hospital approved the protocol.

Methods

Studies were performed using a 148-channel Neuromagnetometer (4D Neuroimaging WH2500), a helmet-shaped device covering the entire adult head, except the face. The individual sensors were SQUID (super conducting quantum interference device) magnetometers. Migraine patients experiencing headache and/or neurological symptoms of aura (visual disturbances: scotoma, phosphenes, fortification spectra; unilateral numbness or weakness of the arm) at the time of the study were monitored for 15-20 min as their symptoms permitted. Migraine subjects not experiencing headaches and controls were monitored for 18 min during presentation of visual stimulus.

The neuromagnetometer was positioned to enclose the head holder and subject's head within the sensor array helmet. The visual stimulus pattern was back-projected on a translucent white projection screen. A black and white circular checkerboard pattern reversal stimulus was chosen to excite edge detection (linear) neurons throughout the visual field rather than color-specific neurons primarily confined to cortex associated with central vision. The computer-generated alternating pattern (implementation software, Matlab version 5.3R11 for Windows 95/98) was projected into the shielded room by a Promax Desktop Projector (Model 5950, Lumens 1250) and onto a large mirror tilted $45^{\circ}$ up. In turn this reflected the image onto another mirror, also tilted at $45^{\circ}$, towards a translucent white projection screen. The image was viewed in a mirror placed above the subject (the center of the mirror was 14 inches $(30 \mathrm{~cm})$ above the face). This mirror was also tilted $45^{\circ}$ toward the translucent white projection screen. The stimulus pattern was a radial checkerboard that subtended $50^{\circ}$ of visual angle at the viewing distance. The check size was $5^{\circ}$ in the radial direction and the stimulus pattern alternated black and white at $8 \mathrm{~Hz}$. Each subject was asked to lie still with eyes open and to focus on a black dot (target) in the center of the oscillating image on the screen. No stimulus pattern was presented to those patients experiencing spontaneous aura, instead they fixed their gaze on a spot on the ceiling.
Data processing

The data were analyzed for DC MEG shifts such as those associated with propagating wave fronts of neuronal depolarization. Magnetic fields encircle neuronal ionic current flow, such that the patterns of magnetic fields enter and exit the skull surface and can be measured with a large array neuromagnetometer system. In the magnetic field contour plots in this article, magnetic fields exiting the skull surface were color-coded red and those entering were color-coded blue.

\section{Statistical analysis}

Fisher's exact tests were used to compare the migraine and control groups for binary variables: sex, DC shifts (yes/no), headache induced (yes/no). A two-sample $t$ test was also used to compare the two groups for age. Additional analyses (Wilcoxon's two sample test with exact $p$ values) were used within the migraine group to compare the magnitude of the DC shift between subjects with and without induced headaches, and between men and women. Wilcoxon's tests were used in place of two sample $t$ tests for these variables because of the small sample sizes and the unequal variances between men and women.

\section{Results}

The three groups were of similar age (spontaneous migraine, $48 \pm 11$ years; visually induced migraine group, $44 \pm 9$ years; control group, $38 \pm 6$ years) and sex distribution (spontaneous migraine group, $50 \%$ women; visually induced migraine group, $62.5 \%$ women; control group, $83.3 \%$ women).

All spontaneous and induced migraine with aura $(\mathrm{M}+\mathrm{A})$ patients experienced visual aura. The eight visually induced $\mathrm{M}+\mathrm{A}$ patients experienced visual symptoms similar to their spontaneous aura. None of the six visually stimulated controls experienced aura ( $p=0.003$, Fisher's exact test). No patient with spontaneous aura experienced headache during the study but all developed headache after the aura ceased and measurements were completed (Table 1). Headache occurred in $5(62 \%)$ of the 8 visually stimulated $\mathrm{M}+\mathrm{A}$ patients during the study but none of the 6 controls developed headache ( $p=0.031$, Fisher's exact test) (Table 2 ).

Typical MEG recordings are shown in Figs. 1-3. Slow DC MEG signal shifts were observed in all spontaneous and visually stimulated $\mathrm{M}+\mathrm{A}$ patients but in none of the visually stimulated controls ( $p=0.003$, Fisher's exact test). There were statistically significant differences $(p=0.0006$, Wilcoxon's) between the DC MEG field amplitude of visually stimulated $\mathrm{M}+\mathrm{A}$ patients (mean amplitude of shifts, $32 \pm 19$ pT, Fig. 3) compared to normal controls ( $<9$ pT, Fig. 1). Difference in the DC MEG field amplitude between the 
Table 1 Spontaneous migraine aura

\begin{tabular}{lcccccc}
\hline Subject & Age, years & Sex & DC shifts & Maximum magnitude, pT & & \multicolumn{2}{c}{ Headache } \\
\cline { 5 - 7 } & & & & During study & Later \\
1 & 49 & F & Yes & 11 & No & Yes \\
2 & 64 & M & Yes & 22 & No & No \\
3 & 42 & F & Yes & 13 & No & Yes \\
4 & 38 & M & Yes & 20 & Yes \\
\hline
\end{tabular}

Table 2 Induced migraine aura

\begin{tabular}{|c|c|c|c|c|c|c|}
\hline Subjects & Age, years & Sex & DC shifts & Maximum magnitude, $\mathrm{pT}$ & Start time, s & Headache \\
\hline \multicolumn{7}{|c|}{ Migraine with aura } \\
\hline 1 & 32 & $\mathrm{~F}$ & Yes & 54 & 150 & Yes \\
\hline 2 & 34 & $\mathrm{~F}$ & Yes & 27 & 110 & Yes \\
\hline 3 & 38 & M & Yes & 12 & 540 & Yes \\
\hline 4 & 40 & M & Yes & 11 & 350 & No \\
\hline 5 & 47 & $\mathrm{~F}$ & Yes & 27 & 200 & Yes \\
\hline 6 & 52 & M & Yes & 13 & 200 & No \\
\hline 7 & 52 & $\mathrm{~F}$ & Yes & 54 & 50 & No \\
\hline 8 & 54 & $\mathrm{~F}$ & Yes & 54 & 520 & Yes \\
\hline \multicolumn{7}{|l|}{ Controls } \\
\hline 9 & 26 & M & No & $<9$ & - & No \\
\hline 10 & 36 & $\mathrm{~F}$ & No & $<9$ & - & No \\
\hline 11 & 37 & $\mathrm{~F}$ & No & $<9$ & - & No \\
\hline 12 & 40 & $\mathrm{~F}$ & No & $<9$ & - & No \\
\hline 13 & 43 & $\mathrm{~F}$ & No & $<9$ & - & No \\
\hline 14 & 43 & $\mathrm{~F}$ & No & $<9$ & - & No \\
\hline
\end{tabular}

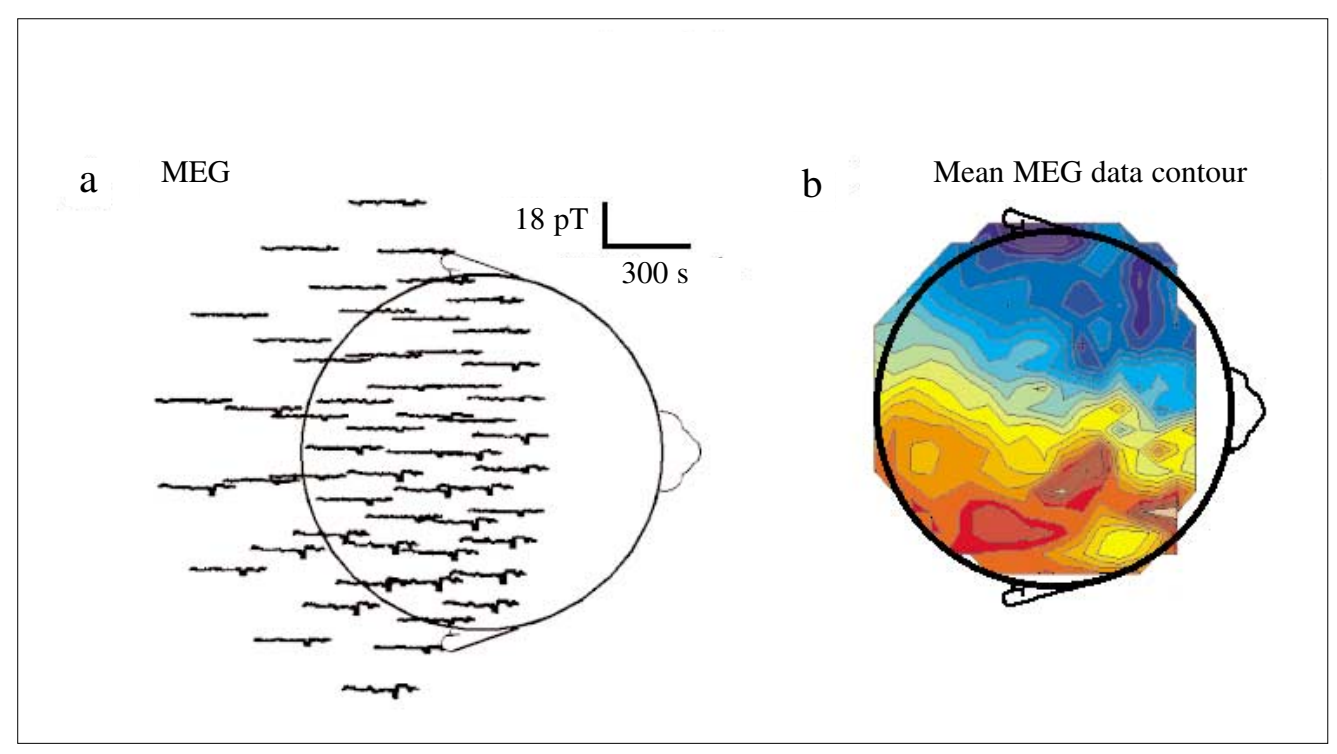

Fig. 1 a, b Control subject. a DC MEG data from normal control subject during visual stimulation. Total time interval is $300 \mathrm{~s}$. The channels at the back of the head have been laid out flat for ease of viewing the data. b Data contour average over the first 200 s. Red corresponds to points where the magnetic field exits the subject's head and blue corresponds to points where the magnetic field enters. No DC shifts are present 


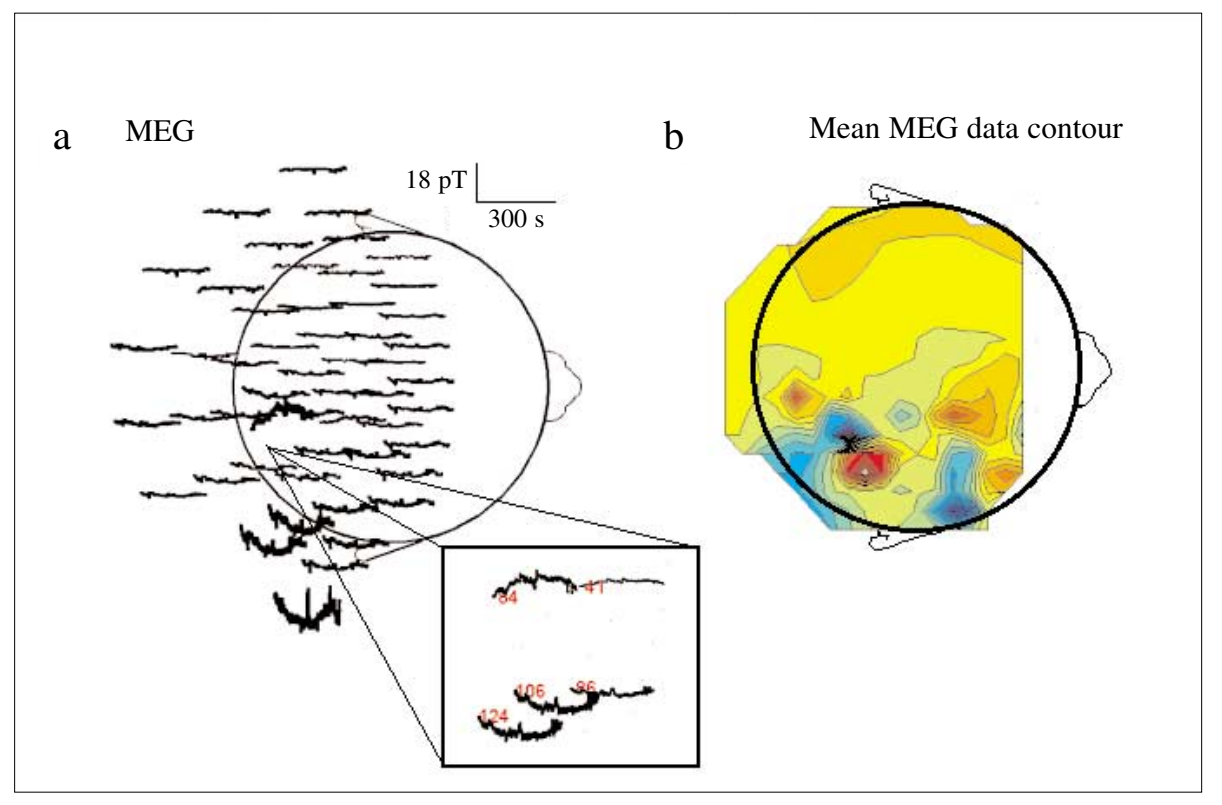

Fig. 2 a, b Spontaneous aura patient. a DC MEG data collected during spontaneous visual aura in a migraine patient. Total time interval is $300 \mathrm{~s}$. b Data contour average over the first $200 \mathrm{~s}$. An average of the first $200 \mathrm{~s}$ of data is displayed showing neuronal activity in the primary visual cortex as well as in the right occipito-temporal cortex

Fig. 3 a, b Induced aura patient. a DC MEG data collected during visually induced migraine aura. Total time interval is $300 \mathrm{~s}$. b Data contour average over the first $200 \mathrm{~s}$

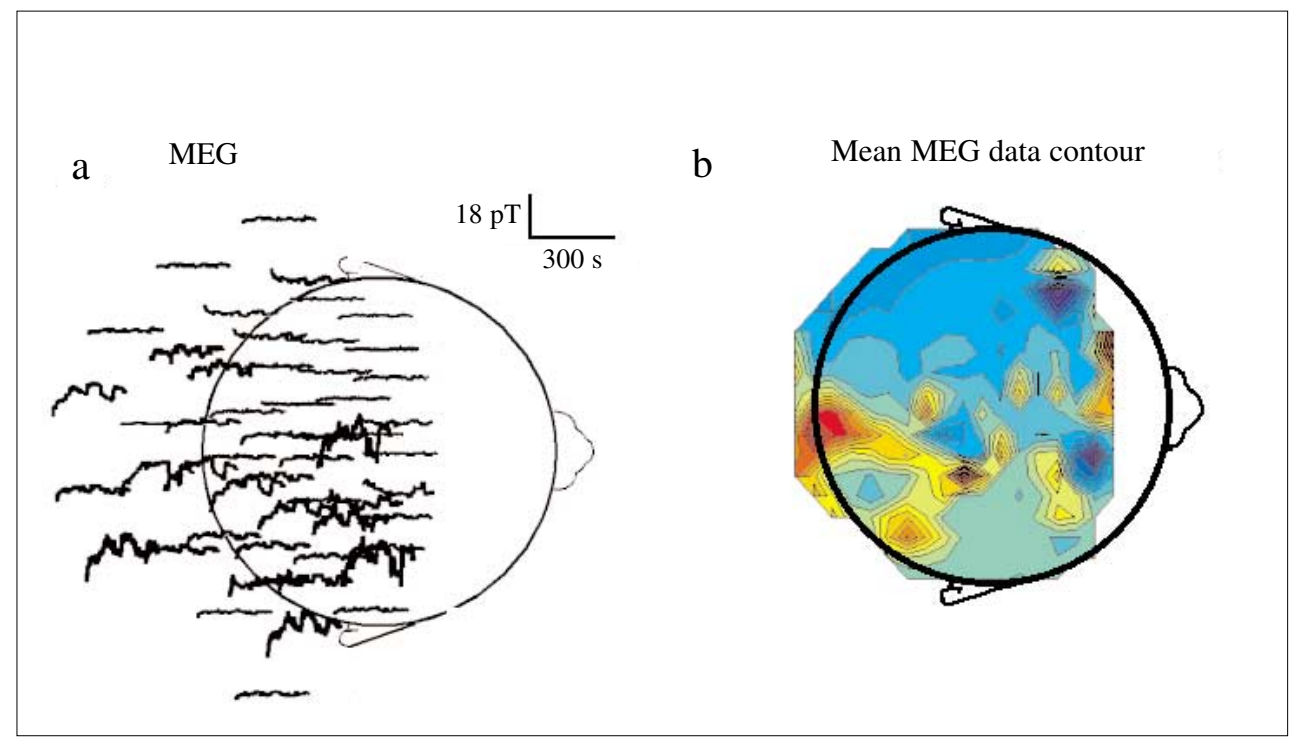

spontaneous migraine patients and visually stimulated controls was significant ( $p=0.0048$, Wilcoxon's) but not between the two migraine groups ( $p=0.25$, Wilcoxon's). The difference in magnitude of the DC shifts was not significant between visually induced migraine patients who experienced headache and those who did not $(34.8 \pm 18.6$ pT vs. $26.0 \pm 24.3$ pT, $p=0.553$, Wilcoxon's two sample test).

Figure 1 displays the occipital MEG channels collected during $300 \mathrm{~s}$ visual stimulation of a control subject. Data were collected for $18 \mathrm{~min}$ and normalized. No DC MEG shifts were recorded. Small spikes (2-5 s shifts from baseline) in the recording are an artifact of patient movement. Figure 2 displays the MEG data of a subject with spontaneous aura. Data were collected approximately $3 \mathrm{~min}$ after aura started. DC MEG signals of large amplitude were recorded from the right hemisphere. Concurrent negative and positive deflections indicated neuroelectric activity occurring under these channels. Figure 3 displays the MEG data of a subject with visually induced aura. Shifts were the same as seen with spontaneous aura.

\section{Discussion}

DC MEG shifts are a direct measurement of neuronal excitation so that MEG should provide more direct knowledge of neuroelectric events such as SCD. Our results demon- 
strate that similar DC MEG field shifts arise during spontaneous and visually induced migraine aura, resembling those arising from SCD crossing a sulcus in animal models $[12,13]$. Furthermore, waveform analysis of the MEG fields observed during migraine aura agree with theoretical predictions from SCD crossing the convolutions of the cortical surface $[8,13]$. The multiphasic form of the MEG waves during aura suggests an extended propagating wave of excitation rather than a local static source on the cortex, strongly supportive of SCD. Waves of neuronal excitation in convoluted brain cortex generate complex MEG field patterns due to propagation in different directions across multiple sulci. Thus, simple equivalent current dipole calculations cannot be applied to the data. Calculating the rate of SCD propagation is difficult and therefore represents, a disadvantage of MEG over the lower resolution blood flow techniques

The model of visually induced migraine aura and headache has been established and refined in our laboratory $[4,16]$. Typified by the present results, when subjects with a natural and reproducible history of migraine attacks induced by visual stress are selected, the success rates for visually induced attacks in our experimental setting are high. In a previously published functional MRI-BOLD study of migraine patients, albeit using a different checkerboard-patterned visual stimulus but delivered at an identical rate, we induced aura and headache in approximately $50 \%$ of patients [4]. Visual stimulation was associated with immediate neu- ronal activation in occipital cortex, though not confined to primary visual cortex. Within similar time delays to the current study, the visual stress initiated spreading suppression of the initial activation at rates compatible with experimental SCD. Multiple events, attributed to an SCD-like phenomenon, were evoked from different regions of the occipital cortex bilaterally. MEG results, using a visual stimulus designed to activate primary visual cortex alone, replicated and, by their more direct nature, extended these findings. Our recordings confirmed the multifocality of neuronal excitation, and the DC MEG shifts that arise from these sites.

Spontaneous auras were associated with DC MEG shifts that emanated from less widespread regions of occipital cortex than those excited by visual stimulation, although still from regions beyond the primary visual cortex. These differences seem best related to the visual stress used to induce the latter. Nevertheless, the visual stimulus of our MEG study was designed to cause physiological activation of primary visual cortex alone, as opposed to the more widespread cortical activation of the stimulus used for our fMRIBOLD study [4]. Interpreted together, the findings from both studies are compatible with hyperexcitability of widespread regions throughout occipital cortex providing the susceptibility for triggering SCD and aura in migraine sufferers [16-18].

Acknowledgements This study was supported by NIH/NINDS grants RO1-NS30914 to NT and P50-NS32399 to KMAW.

\section{References}

1. Leão AAP (1994) Spreading depression of activity in the cerebral cortex. J Neurophysiol 7:379-390

2. Milner PM (1958) Note on possible correspondence between the scotomas of migraine and spreading depression of Leao. Electroencephalogr Clin Neurophysiol 10:705

3. Lauritzen M (1994) Pathophysiology of the migraine aura; the spreading depression theory. Brain 117:199-210

4. Cao Y, Welch KMA, Aurora S, Vikingstad E (1999) Functional MRIBold of visually triggered headache in patients with migraine. Arch Neurol 56:548-554

5. Hansen AJ, Zeuthen T (1981) Extracellular ion concentration during spreading depression and ischemia in the rat brain cortex. Acta Physiol Scand 113:437-445
6. Nicholson C, Kraig RP (1981) The behavior of extracellular ions during spreading depression. In: Zeuther T (ed) The application of ion selective microelectrodes Elsevier, Amsterdam, pp 217-238

7. Hämäläinen M, Hari R, Ilmoniemi RJ et al (1993) Magnetoencephalography - theory, instrumentation, and applications to noninvasive studies of the working human brain. Rev Modern Physics 65:413-497

8. Okada YC, Lauritzen M, Nicholson C (1988) Magnetic fields associated with SD: a model for the detection of migraine. Brain Res 442:185-190

9. Okada YC (1990) Magnetoencephalography as a non-invasive tool for electrophysiological characterization of auras in classic migraine. In: Sato S (ed) Magnetoencephalography. Raven, New York, pp 133-140 (Advances in Neurology, vol. 54)
10. Barkley GL, Tepley N, Nagel-Leiby S et al (1990) Magnetoencephalographic studies of migraine. Headache 30:428-434

11. Gardner-Medwin AR, Tepley N, Barkley GL et al (1991) Magnetic fields associated with spreading depression in anaesthetized rabbits. Brain Res 540:153-158

12. Bowyer SM, Okada YC, Papuashvili N et al (1999) Analysis of MEG signals of spreading cortical depression with propagation constrained to a rectangular cortical strip: I. Lissencephalic rabbit model. Brain Res 843:71-78

13. Bowyer SM, Tepley N, Papuashvili N et al (1999) Analysis of MEG signals of spreading cortical depression with propagation constrained to a rectangular cortical strip: II. Gyrencephalic swine model. Brain Res 843:79-86 
14. - (1988) Classification and diagnostic criteria for headache disorders, cranial neuralgias, and facial pain. Headache Classification Committee of the International Headache Society. Cephalalgia 8[Suppl 7]:1-96
15. Moran JE, Tepley N (2000) Two dimensional inverse imaging (2DII) of current sources in magnetoencephalography. Brain Topogr 12:201-217

16. Aurora SK, Cao Y, Bowyer S, Welch KMA (1999) The occipital cortex is hyperexcitable in migraine: experimental evidence. Headache 39:469-476
17. Aurora SK, Ahmad BK, Welch KM et al (1998) Transcranial magnetic stimulation confirms hyperexcitability of occipital cortex in migraine. Neurology 50:1111-1114

18. Welch KMA, D'Andrea G, Tepley N et al (1990) The concept of migraine as a state of central neuronal hyperexcitability. Neurol Clin 8:817-818 